\title{
Viscosity and Density of Water + Ethyl Acetate + Ethanol Mixtures at 298.15 and 318.15 K and Atmospheric Pressure
}

\author{
Rui M. Pires, Henrique F. Costa, Abel G. M. Ferreira, and Isabel M. A. Fonseca* \\ Departamento de Engenharia Química, Universidade de Coimbra, Pólo II, Pinhal de Marrocos, 3030-290 Coimbra, Portugal
}

\begin{abstract}
The viscosities and densities of the ternary mixtures water + ethyl acetate + ethanol and their constituent binaries have been measured at 298.15 and $318.15 \mathrm{~K}$ and atmospheric pressure. The excess molar volumes, $V_{\mathrm{m}}^{\mathrm{E}}$, and viscosity deviations, $\Delta \eta$, were calculated from density and viscosity, respectively. A rational function due to Myers and Scott was used to describe the composition dependence of these properties. To describe the ternary system, binary pair additivity and the Pando et al. rational functions for the ternary contributions $\left(\Delta \eta_{\mathrm{T}}\right.$ and $\left.V_{\mathrm{T}}^{\mathrm{E}}\right)$ were considered.
\end{abstract}

\section{Introduction}

The extensive information on the thermodynamic and transport properties of liquid mixtures is needed in multiple industrial process areas such as fluid flow and heat and mass transfer and also for the development of theoretical research on molecular interactions in solutions. The systems alkanol + alkanoate are of great interest from a theoretical point of view because in mixing processes the breaking of $\mathrm{H}$-bonded structures of the alcohol occurs and the formation of new H-bonded molecular species between the alcohol and the ester takes place. ${ }^{1}$ Ternary mixtures are the easiest multicomponent systems to treat experimentally and represent the appropriate ground for the application of correlation and prediction models.

The present work is part of a research program which has been developed in our laboratory that aims to provide density, viscosity, and surface tension data for ternary and binary subsystems of the type water + alkanoate + alkanol at different temperatures. ${ }^{2,3}$ These data are particularly important in the pharmaceutical, food, and flavor industries.

We report in this paper the density, $\rho$, and the dynamic viscosity, $\eta$, for the ternary mixtures of water + ethyl acetate + ethanol and its binary constituents at 298.15 and $318.15 \mathrm{~K}$ and atmospheric pressure.

The performance of different analytical $V_{\mathrm{m}}^{\mathrm{E}}$ models for the correlation and for the prediction have been studied in the literature. ${ }^{4}$ The conclusion is that the models including polynomial rational functions give low standard deviations. These kind of functions found a wide acceptance and have been applied to the excess enthalpy, $H^{\mathrm{E}, 5}$, and excess Gibbs energy, $G^{\mathrm{E}} \cdot{ }^{6}$ The polynomial rational functions were first proposed by Myers and Scott. ${ }^{7}$ They have been used in this study together with the rational functions of Pando et al. ${ }^{8}$ to correlate excess molar volume and viscosity deviation.

\section{Experimental Techniques}

Materials. Tridistilled water was used. Ethanol (Cromasolv, HPLC grade) was supplied with a mass fraction purity better than 0.998. Ethyl acetate was supplied by Riedel de Häen with a stated purity better than 0.997 . The measured viscosities and

* To whom correspondence should be addressed. E-mail: fonseca@eq.uc.pt. Phone: +351-239 798700. Fax: +351-239 798703
Table 1. Densities, $\rho$, and Viscosities, $\eta$, of the Pure Components at 298.15 and $318.15 \mathrm{~K}$

\begin{tabular}{|c|c|c|c|c|}
\hline \multirow[b]{2}{*}{ substance } & \multicolumn{2}{|c|}{$\rho / g \cdot \mathrm{cm}^{-3}$} & \multicolumn{2}{|c|}{$\eta / \mathrm{mPa} \cdot \mathrm{s}$} \\
\hline & exptl & literature & exptl & literature \\
\hline \multicolumn{5}{|c|}{$T=298.15 \mathrm{~K}$} \\
\hline water & - & $0.99704^{a}$ & - & $0.8902^{b}$ \\
\hline ethyl acetate & 0.89444 & $0.89445^{c}$ & 0.427 & $0.4260^{d}$ \\
\hline ethanol & 0.78510 & $0.78510^{e}$ & 1.102 & \\
\hline & & $0.78513^{g}$ & & $1.105^{f}$ \\
\hline air & & $1.169 \cdot 10^{h}$ & & \\
\hline \multicolumn{5}{|c|}{$T=318.15 \mathrm{~K}$} \\
\hline water & - & $0.99017^{a}$ & - & $0.5961^{b}$ \\
\hline ethyl acetate & 0.86997 & $0.86990^{c}$ & 0.356 & $0.346^{i}$ \\
\hline $\begin{array}{l}\text { ethanol } \\
\text { air }\end{array}$ & 0.76842 & $\begin{array}{l}0.76811^{j} \\
1.095 \cdot 10^{h}\end{array}$ & 0.749 & $0.7461^{j}$ \\
\hline
\end{tabular}

densities of the three components together with values found in the literature are presented in Table 1.

Measurements. Viscosities were measured by the Haake Falling Ball viscosimeter (Hoppler design), calibrated with tridistilled water. An electronic digital stopwatch, with an uncertainty of $\pm 1 \cdot 10^{-2} \mathrm{~s}$, was used to measure the falling time of the ball. A borosilicate ball with a $15.81 \mathrm{~mm}$ diameter and with a density of $2.2186 \mathrm{~g} \cdot \mathrm{cm}^{-3}$ was used.

Densities were measured in an Anton Paar DMA 60 digital vibrating tube densimeter, with a DMA 602 measuring cell. Air and pure water were used for the calibration of the densimeter. The temperature maintenance and control were performed using an Ultraterm P Selecta thermostatic water bath, which has a temperature uncertainty of $\pm 1 \cdot 10^{-2} \mathrm{~K}$. In the case of density measurements, the Pt resistance thermometer (calibrated against a standard mercury thermometer, graduated in $0.01 \mathrm{~K}$, certified by NPL, U.K.) was placed inside the vibrating tube densimeter to find the actual temperature of the measurements.

Mixtures were prepared by mass using a Mettler AT 200 balance with a precision of $\pm 1 \cdot 10^{-5} \mathrm{~g}$. The uncertainty of the mole fraction is estimated to be of the order $\pm 1 \cdot 10^{-4}$. The measurement of both properties was accomplished using the following methodology. A binary mixture was used as a starting point of measurements in the water (1) + ethanol (3) or ethyl acetate (2) + ethanol (3) systems. The ternary data were 

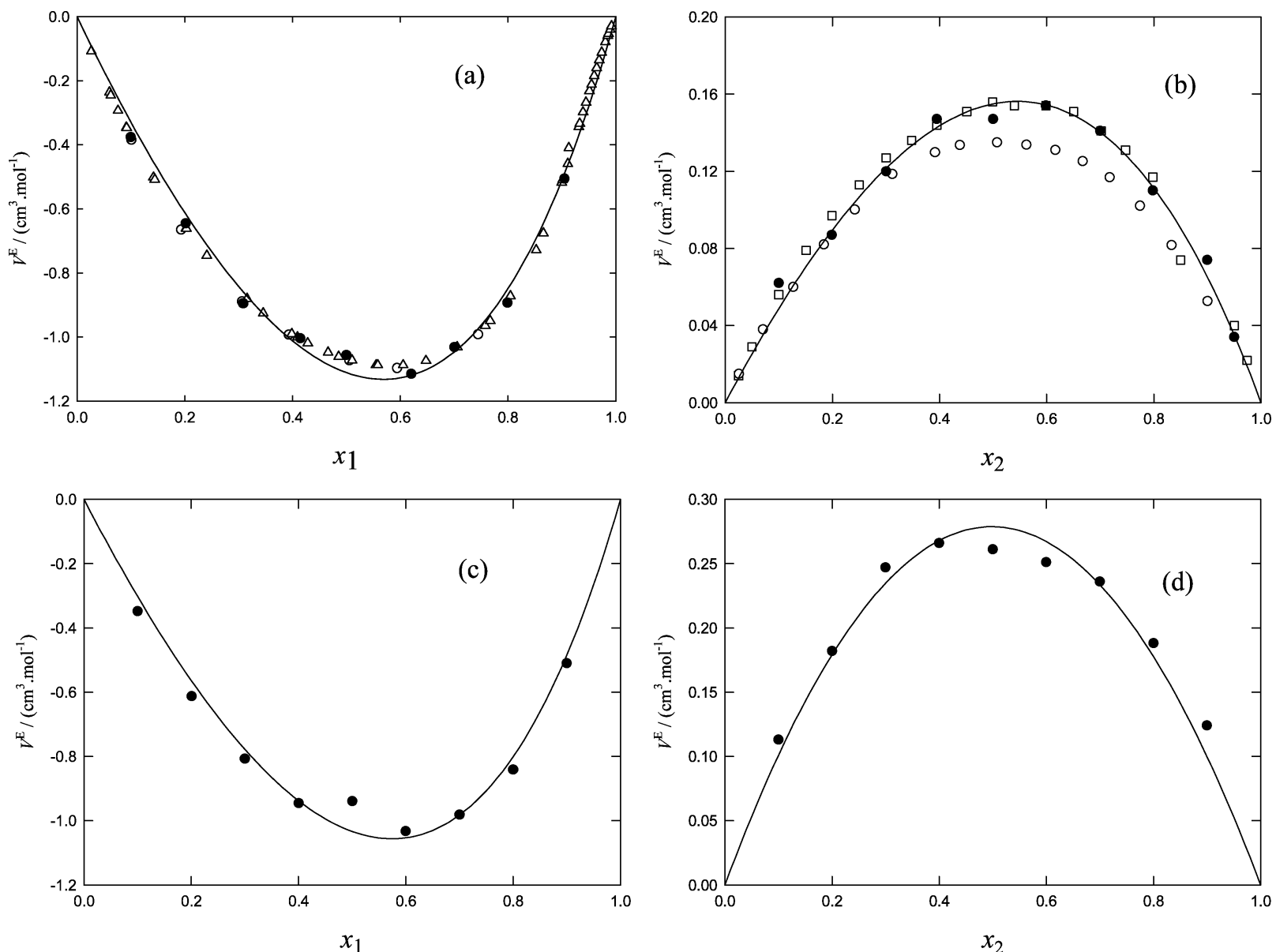

Figure 1. Excess molar volume, $V_{\mathrm{m}}^{\mathrm{E}}$, of the binary systems at $298.15 \mathrm{~K}$ as a function of the mole fractions: (a) water (1) + ethanol (3) and (b) ethyl acetate (2) + ethanol (3). (a) Experimental data: $\bigcirc$, this work; $\bigcirc$, Arce et al. ${ }^{14,18} \triangle$, Benson et al. ${ }^{19}$ (b) Experimental data: $\bigcirc$, this work; $\bigcirc$, Ortega et al.; ${ }^{20} \square$, Hu et al. ${ }^{21}$ At $318.15 \mathrm{~K}$ : (c) water (1) + ethanol (3) and (d) ethyl acetate (2) + ethanol (3). (c) Experimental data:

, this work. The curves were calculated with eq 4 .

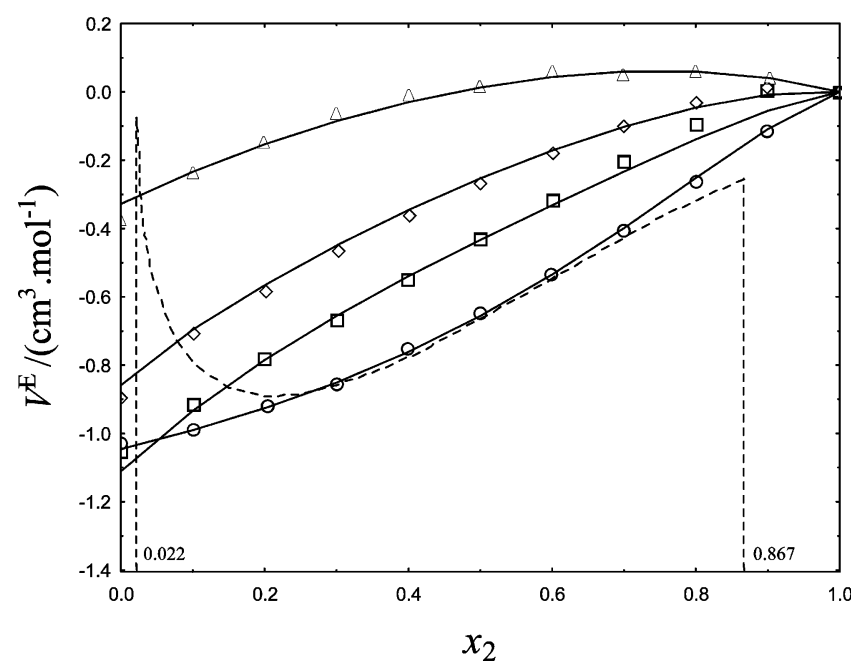

Figure 2. Excess molar volume, $V_{\mathrm{m}}^{\mathrm{E}}$, of the system water (1) + ethyl acetate (2) + ethanol (3) at $298.15 \mathrm{~K}$ following lines of constant $z=x_{3} / x_{1}$ The symbols represent experimental data, and the lines represent the calculated values: $\bigcirc, z=0.43 ; \square, z=1.00 ; \diamond, z=2.33 ; \Delta, z=9.00 ;-$, eqs 5 and $6 ;---$, binodal curve. 22,23

obtained following lines of constant molar fraction ratio of $z=$ $x_{3} / x_{1}$ and $z=x_{3} / x_{2}$, respectively.

Uncertainties. Densities were measured with an uncertainty of $\pm 1 \cdot 10^{-5} \mathrm{~g} \cdot \mathrm{cm}^{-3}$. Having in mind that the error in the excess molar volume, $V_{\mathrm{m}}^{\mathrm{E}}$, is determined by the uncertainties in mole

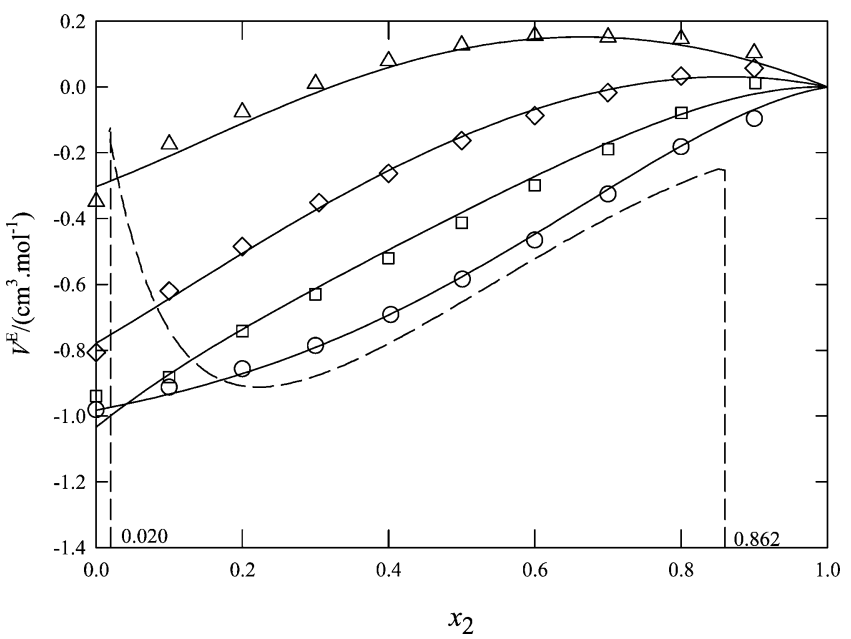

Figure 3. Excess molar volume, $V_{\mathrm{m}}^{\mathrm{E}}$, of the system water $(1)+$ ethyl acetate (2) + ethanol (3) at $318.15 \mathrm{~K}$ following lines of constant $z=x_{3} / x_{1}$. The symbols represent experimental data, and the lines represent calculated values: $\bigcirc, z=0.43 ; \square, z=1.00 ; \diamond, z=2.33 ; \Delta, z=9.00 ;-$, eqs 5 and 6 ; - - -, binodal curve. ${ }^{22,23}$

fraction and density, the maximum error in $V_{\mathrm{m}}^{\mathrm{E}}$ resulting from the propagation law of errors is $\pm 1 \cdot 10^{-3} \mathrm{~cm}^{3} \cdot \mathrm{mol}^{-1}$. Taking into consideration the uncertainties in the measured time and in the density, the experimental uncertainty in the viscosity is $\pm 1 \cdot 10^{-3} \mathrm{mPa} \cdot \mathrm{s}$. 

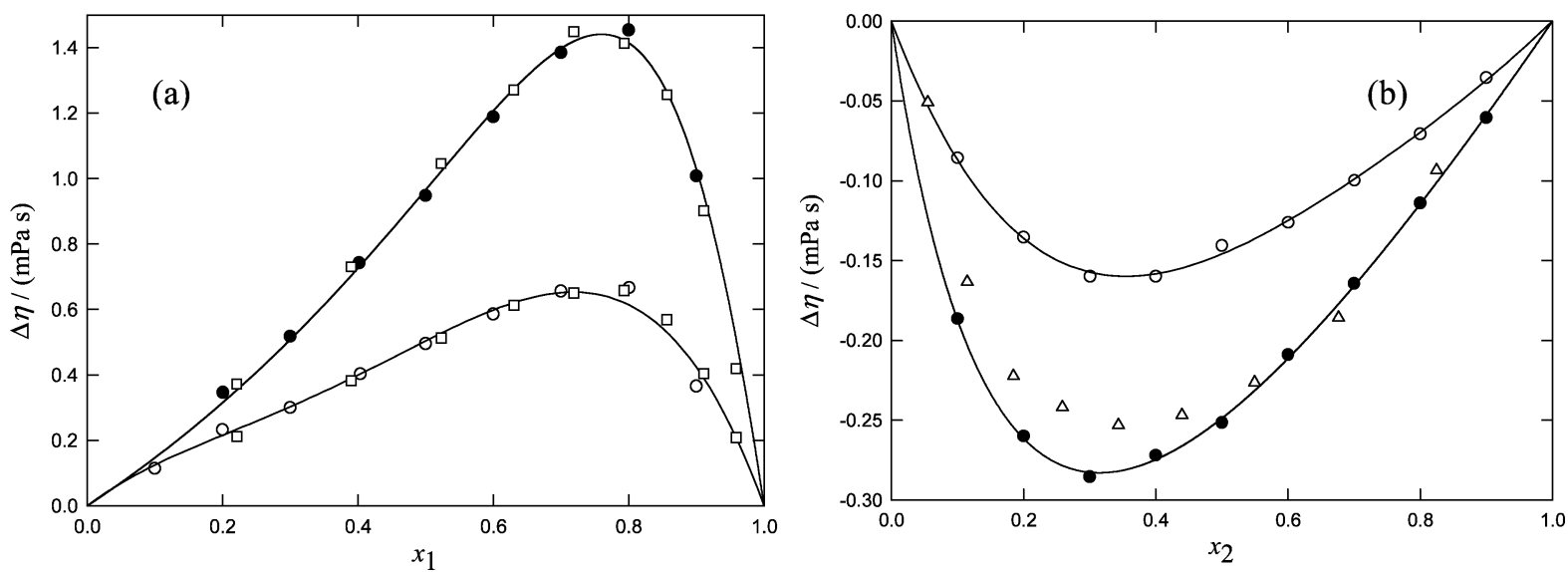

Figure 4. Viscosity deviations, $\Delta \eta$, of the binary systems at $298.15 \mathrm{~K}$ and $318.15 \mathrm{~K}$ as a function of the mole fractions: (a) water (1) + ethanol (3) and (b) ethyl acetate (2) + ethanol (3). The symbols refer to experimental data: $\bigcirc$, this work at $298.15 \mathrm{~K}$; $\bigcirc$, this work at $318.15 \mathrm{~K}$; $\square$, Vargaftik; ${ }^{15} \Delta$, Nikam et al. ${ }^{11}$ at $298.15 \mathrm{~K}$. The curves are calculated with eq 4.

Table 2. Experimental Densities, $\rho$, and Excess Molar Volumes, $V_{\mathrm{m}}^{\mathrm{E}}$, for the System Water (1) + Ethyl Acetate (2) + Ethanol (3) and Its Binary Constituents at 298.15 K and Atmospheric Pressure

\begin{tabular}{|c|c|c|c|c|c|c|c|}
\hline & & $\rho$ & $V_{\mathrm{m}}^{\mathrm{E}}$ & & & $\rho$ & $V_{\mathrm{m}}^{\mathrm{E}}$ \\
\hline$x_{1}$ & $x_{2}$ & -3 & 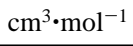 & $x_{1}$ & $x_{2}$ & $g$ & 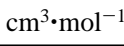 \\
\hline 0269 & 0.9731 & 0.89532 & -0.039 & 2091 & 0.3036 & 0.856 & -0.46 \\
\hline & & & & & & & \\
\hline 0.0722 & 0.9278 & & -0. & 00 & 0.5005 & 0.87056 & -0.267 \\
\hline 0892 & 0.9108 & & -0 . & 0 & 0.6 & 23 & \\
\hline & 0.8955 & & -0. & & & & -0.099 \\
\hline 1249 & 0.8751 & 9950 & -0 & 600 & 0.8002 & 85 & -0.032 \\
\hline 9051 & 0 & 6 & -0 & & 0.9001 & 0.89010 & 0.014 \\
\hline 7002 & 0 & & -0 . & & 0.1 & & -0.239 \\
\hline 10 & 0 & & -1 . & & 0.2 & & -0 \\
\hline & 0 & & -1 . & & & & -0.069 \\
\hline 1 & 0 & & -1 . & & & & -0.011 \\
\hline & 0 & & -1. & & & & 0.010 \\
\hline 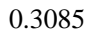 & 0 & & & & & & .055 \\
\hline 2018 & 0 & & -0 . & & & & .048 \\
\hline 00 & 0 & & -0 & & & & .057 \\
\hline & 01 & 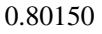 & & & & & 0.038 \\
\hline & & & & & & & -0.274 \\
\hline & & & & & & & -0.561 \\
\hline & & & & & & & -0 \\
\hline & 0.5 & 0 & 7 & 9 & & & -0.953 \\
\hline & & & & & & & -1.003 \\
\hline & & & & & & & 27 \\
\hline & & & & & & & -1.006 \\
\hline & 0 & & & & 0.0 & & -0.778 \\
\hline & & & & & & & -0.454 \\
\hline & & & & & & & \\
\hline & & & & & & & -0 \\
\hline & & & & & & & 91 \\
\hline 2 & & & & & & & -0.837 \\
\hline & & & & & & & -0.914 \\
\hline & & & & & & & -0 \\
\hline & & & & & & & -0.890 \\
\hline .1401 & 0.8000 & & -0 & 02 & 0.0 & & -0.704 \\
\hline & & & & & & & \\
\hline & & & & & & & \\
\hline & & & & & & & -0 . \\
\hline 3 & 0.29 & & -0 & & 0.3500 & & -0.614 \\
\hline 3002 & 0.3999 & & -0. & 0.4000 & 0.3000 & & -0.786 \\
\hline & & & & & & & \\
\hline & & & & & & & \\
\hline & & & & & & & -0.127 \\
\hline & & & -0.095 & & & & -0.358 \\
\hline 0 & 0.9001 & & 0.001 & 0.3059 & 0.4859 & 62 & -0.582 \\
\hline & & & -0.707 & 0.4000 & 0.4200 & 0.89852 & -0.728 \\
\hline & & & & & & & \\
\hline
\end{tabular}

\section{Experimental Results and Discussion}

In Tables 2 and 3, the densities, $\rho$, are listed as a function of the composition at the two temperatures. In the same tables,
Table 3. Experimental Densities, $\rho$, and Excess Molar Volumes, $V_{\mathrm{m}}^{\mathrm{E}}$, for the System Water (1) + Ethyl Acetate (2) + Ethanol (3) and Its Binary Constituents at 318.15 $\mathrm{K}$ and Atmospheric Pressure

$x_{1} \quad x_{2} \quad \frac{\rho}{\mathrm{g} \cdot \mathrm{cm}^{-3}} \frac{V_{\mathrm{m}}^{\mathrm{E}}}{\mathrm{cm}^{3} \cdot \mathrm{mol}^{-1}} \quad x_{1} \quad x_{2} \frac{\rho}{\mathrm{g} \cdot \mathrm{cm}^{-3}} \frac{V_{\mathrm{m}}^{\mathrm{E}}}{\mathrm{cm}^{3} \cdot \mathrm{mol}^{-1}}$

\begin{tabular}{lllllllll}
\hline 0.0249 & 0.9751 & 0.87081 & -0.033 & 0.1802 & 0.4000 & 0.84183 & -0.262
\end{tabular}

$\begin{array}{lllllllll}0.0524 & 0.9476 & 0.87180 & -0.072 & 0.1501 & 0.5000 & 0.84759 & -0.163\end{array}$

$\begin{array}{lllllllll}0.0766 & 0.9234 & 0.87273 & -0.108 & 0.1201 & 0.5999 & 0.85282 & -0.087\end{array}$

$\begin{array}{lllllllll}0.1003 & 0.8997 & 0.87394 & -0.171 & 0.0901 & 0.7000 & 0.85744 & -0.017\end{array}$

$\begin{array}{lllllllll}0.1255 & 0.8745 & 0.87508 & -0.216 & 0.0601 & 0.8000 & 0.86169 & 0.033\end{array}$

$\begin{array}{llllllll}0.9000 & 0 & 0.95247 & -0.510 & 0.0300 & 0.9000 & 0.86570 & 0.057\end{array}$

$\begin{array}{llllllllll}0.8001 & 0 & & 0.91919 & -0.841 & 0.0902 & 0.1000 & 0.79380 & -0.174\end{array}$

$\begin{array}{llllllllll}0.7001 & 0 & 0.88876 & -0.981 & 0.0802 & 0.2000 & 0.80606 & -0.076\end{array}$

$\begin{array}{lllllllll}0.5998 & 0 & & 0.86331 & -1.033 & 0.0702 & 0.3000 & 0.81683 & 0.010\end{array}$

$\begin{array}{lllllllll}0.5003 & 0 & & 0.84031 & -0.939 & 0.0601 & 0.4000 & 0.82643 & 0.079\end{array}$

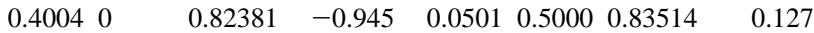

$\begin{array}{lllllllll}0.3001 & 0 & & 0.80771 & -0.807 & 0.0401 & 0.6000 & 0.84307 & 0.156\end{array}$

$\begin{array}{lllllllll}0.2010 & 0 & & 0.79360 & -0.613 & 0.0301 & 0.7001 & 0.85051 & 0.151\end{array}$

$\begin{array}{llllllllll}0.1000 & 0 & 0.78053 & -0.348 & 0.0200 & 0.8000 & 0.85721 & 0.146\end{array}$

$\begin{array}{llllllll}0 & 0.1000 & 0.78309 & 0.113 & 0.0100 & 0.9000 & 0.86362 & 0.103\end{array}$

$\begin{array}{lllllllll}0 & 0.2001 & 0.79645 & 0.182 & 0.1000 & 0.0900 & 0.79301 & -0.168\end{array}$

$\begin{array}{lllllllll}0 & 0.3000 & 0.80831 & 0.247 & 0.2010 & 0.0799 & 0.80552 & -0.499\end{array}$

$\begin{array}{lllllllll}0 & 0.4001 & 0.81937 & 0.266 & 0.3000 & 0.0700 & 0.81823 & -0.704\end{array}$

$\begin{array}{lllllllll}0 & 0.5000 & 0.82953 & 0.261 & 0.4000 & 0.0600 & 0.83274 & -0.853\end{array}$

$\begin{array}{lllllllll}0 & 0.6000 & 0.83875 & 0.251 & 0.5004 & 0.0500 & 0.84959 & -0.947\end{array}$

$\begin{array}{lllllllll}0 & 0.7000 & 0.84718 & 0.236 & 0.6001 & 0.0400 & 0.86909 & -0.973\end{array}$

$\begin{array}{lllllllll}0 & 0.8001 & 0.85516 & 0.188 & 0.7004 & 0.0300 & 0.89358 & -0.966\end{array}$

$\begin{array}{lllllllll}0 & 0.8999 & 0.86260 & 0.124 & 0.8000 & 0.0200 & 0.92159 & -0.823\end{array}$

$\begin{array}{lllllllll}0.6300 & 0.1001 & 0.88433 & -0.913 & 0.8997 & 0.0100 & 0.95419 & -0.525\end{array}$

$\begin{array}{llllllll}0.5600 & 0.2000 & 0.88156 & -0.857 & 0.1005 & 0.2698 & 0.81719 & -0.101\end{array}$

$\begin{array}{lllllllll}0.4901 & 0.3000 & 0.87932 & -0.786 & 0.2001 & 0.2402 & 0.82677 & -0.378\end{array}$

$\begin{array}{lllllllll}0.4179 & 0.4031 & 0.87726 & -0.692 & 0.2998 & 0.2102 & 0.83743 & -0.598\end{array}$

$\begin{array}{llllllllll}0.3493 & 0.5010 & 0.87549 & -0.584 & 0.3955 & 0.1815 & 0.84893 & -0.752\end{array}$

$\begin{array}{lllllllll}0.2801 & 0.5999 & 0.87393 & -0.466 & 0.5004 & 0.1500 & 0.86358 & -0.862\end{array}$

$\begin{array}{llllllll}0.2100 & 0.7000 & 0.87242 & -0.326 & 0.5999 & 0.1201 & 0.88016 & -0.907\end{array}$

$\begin{array}{lllllllll}0.1401 & 0.7999 & 0.87112 & -0.182 & 0.6999 & 0.0901 & 0.90041 & -0.884\end{array}$

$\begin{array}{llllllll}0.0699 & 0.9001 & 0.87054 & -0.096 & 0.8000 & 0.0601 & 0.92541 & -0.769\end{array}$

$\begin{array}{lllllllll}0.4504 & 0.0999 & 0.84779 & -0.882 & 0.9000 & 0.0300 & 0.95755 & -0.548\end{array}$

$\begin{array}{lllllllll}0.4004 & 0.2000 & 0.85200 & -0.742 & 0.1000 & 0.4500 & 0.83669 & -0.054\end{array}$

$\begin{array}{lllllllll}0.3504 & 0.2999 & 0.85569 & -0.630 & 0.2028 & 0.3986 & 0.84485 & -0.326\end{array}$

$\begin{array}{llllllll}0.3003 & 0.3999 & 0.85868 & -0.520 & 0.3009 & 0.3496 & 0.85359 & -0.535\end{array}$

$\begin{array}{llllllll}0.2501 & 0.5002 & 0.86118 & -0.413 & 0.4018 & 0.2991 & 0.86405 & -0.710\end{array}$

$\begin{array}{llllllll}0.2002 & 0.6000 & 0.86315 & -0.299 & 0.4996 & 0.2502 & 0.87574 & -0.817\end{array}$

$\begin{array}{lllllllll}0.1501 & 0.7003 & 0.86490 & -0.189 & 0.5998 & 0.2001 & 0.88990 & -0.861\end{array}$

$\begin{array}{lllllllll}0.0998 & 0.8005 & 0.86638 & -0.079 & 0.0993 & 0.6305 & 0.85379 & -0.121\end{array}$

$\begin{array}{llllllll}0.0500 & 0.9018 & 0.86793 & 0.012 & 0.2002 & 0.5599 & 0.85964 & -0.292\end{array}$

$\begin{array}{lllllllll}0.2702 & 0.1000 & 0.81816 & -0.620 & 0.3081 & 0.4844 & 0.86800 & -0.527\end{array}$

$\begin{array}{llllllll}0.2402 & 0.2000 & 0.82732 & -0.485 & 0.4011 & 0.4193 & 0.87610 & -0.671\end{array}$

$\begin{array}{llll}0.2087 & 0.3050 & 0.83533 & -0.351\end{array}$

the excess molar volumes, $V_{\mathrm{m}}^{\mathrm{E}}$, of the mixture were obtained from the measured density by

$$
V_{\mathrm{m}}^{\mathrm{E}}=\rho^{-1}\left(\sum x_{i} M_{i}\right)-\sum x_{i} M_{i} \rho_{i}^{-1}
$$




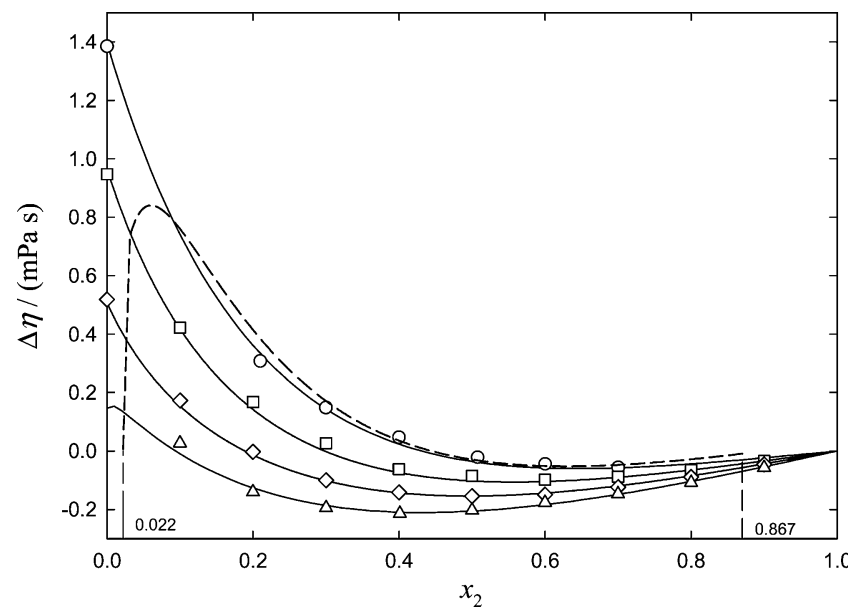

Figure 5. Viscosity, $\Delta \eta$, of the system water (1) + ethyl acetate (2) + ethanol (3) at $298.15 \mathrm{~K}$ following lines of constant $z=x_{3} / x_{1}$. The symbols represent experimental data, and the lines are calculated: $\mathrm{O}, z=$ $0.43 ; \square, z=1.00 ; \diamond, z=2.33 ; \Delta, z=9.00 ;-$, eqs 5 and $6 ;---$, binodal curve. ${ }^{22,23}$

Table 4. Experimental Viscosities, $\eta$, for the System Water (1) + Ethyl Acetate (2) + Ethanol (3) and Its Binary Constituents at 298.15 K and Atmospheric Pressure

\begin{tabular}{lccccc}
\hline & & $\eta$ & & & $\eta$ \\
\cline { 3 - 5 }$x_{1}$ & $x_{2}$ & $\mathrm{mPa} \cdot \mathrm{s}$ & $x_{1}$ & $x_{2}$ & $\mathrm{mPa} \cdot \mathrm{s}$ \\
\hline 0.0258 & 0.9742 & 0.436 & 0.1798 & 0.4001 & 0.653 \\
0.0450 & 0.9550 & 0.444 & 0.1499 & 0.5001 & 0.579 \\
0.0748 & 0.9252 & 0.456 & 0.1199 & 0.6000 & 0.523 \\
0.0853 & 0.9147 & 0.460 & 0.0899 & 0.7001 & 0.487 \\
0.1053 & 0.8947 & 0.468 & 0.0599 & 0.8001 & 0.460 \\
0.1258 & 0.8742 & 0.476 & 0.0300 & 0.9000 & 0.442 \\
0.2009 & 0 & 1.407 & 0.0902 & 0.1000 & 1.042 \\
0.3001 & 0 & 1.558 & 0.0802 & 0.2000 & 0.810 \\
0.4016 & 0 & 1.761 & 0.0701 & 0.3000 & 0.691 \\
0.5001 & 0 & 1.946 & 0.0600 & 0.4012 & 0.605 \\
0.6000 & 0 & 2.166 & 0.0501 & 0.5000 & 0.551 \\
0.7000 & 0 & 2.342 & 0.0401 & 0.6000 & 0.512 \\
0.8000 & 0 & 2.390 & 0.0301 & 0.7000 & 0.476 \\
0.9000 & 0 & 1.923 & 0.0200 & 0.8000 & 0.450 \\
0 & 0.1000 & 0.848 & 0.0100 & 0.9000 & 0.435 \\
0 & 0.2000 & 0.707 & 0.2000 & 0.0800 & 1.132 \\
0 & 0.3000 & 0.614 & 0.3008 & 0.0699 & 1.283 \\
0 & 0.4000 & 0.560 & 0.4000 & 0.0600 & 1.461 \\
0 & 0.5000 & 0.513 & 0.5003 & 0.0500 & 1.654 \\
0 & 0.6000 & 0.488 & 0.6000 & 0.0400 & 1.868 \\
0 & 0.7000 & 0.465 & 0.7001 & 0.0300 & 2.069 \\
0 & 0.8000 & 0.448 & 0.8000 & 0.0200 & 2.119 \\
0 & 0.9000 & 0.434 & 0.9000 & 0.0100 & 1.834 \\
0.5529 & 0.2101 & 1.153 & 0.1002 & 0.2700 & 0.713 \\
0.4900 & 0.3000 & 0.946 & 0.2000 & 0.2400 & 0.821 \\
0.4200 & 0.4000 & 0.791 & 0.3000 & 0.2100 & 0.948 \\
0.3446 & 0.5078 & 0.666 & 0.4002 & 0.1800 & 1.107 \\
0.2800 & 0.6001 & 0.594 & 0.5000 & 0.1500 & 1.268 \\
0.2098 & 0.7002 & 0.529 & 0.6000 & 0.1200 & 1.464 \\
0.1401 & 0.7999 & 0.480 & 0.7000 & 0.0900 & 1.679 \\
0.4499 & 0.1001 & 1.363 & 0.8000 & 0.0600 & 1.844 \\
0.3999 & 0.2000 & 1.052 & 0.9000 & 0.0300 & 1.681 \\
0.3500 & 0.3000 & 0.854 & 0.1001 & 0.4500 & 0.579 \\
0.3002 & 0.3999 & 0.708 & 0.2003 & 0.3999 & 0.661 \\
0.2502 & 0.4999 & 0.628 & 0.3002 & 0.3499 & 0.762 \\
0.2001 & 0.6000 & 0.557 & 0.4001 & 0.3000 & 0.887 \\
0.1501 & 0.7000 & 0.510 & 0.5002 & 0.2499 & 1.027 \\
0.1000 & 0.8000 & 0.477 & 0.6002 & 0.1999 & 1.226 \\
0.0500 & 0.9001 & 0.450 & 0.1003 & 0.6297 & 0.506 \\
0.2697 & 0.1002 & 1.151 & 0.3002 & 0.4898 & 0.646 \\
0.2398 & 0.2001 & 0.914 & 0.4000 & 0.4200 & 0.745 \\
0.2098 & 0.3000 & 0.756 & & & \\
& & & & &
\end{tabular}

where $x_{i}, M_{i}$, and $\rho_{i}$ are the mole fraction, the molecular weight, and the measured density of the pure component $i$, respectively.

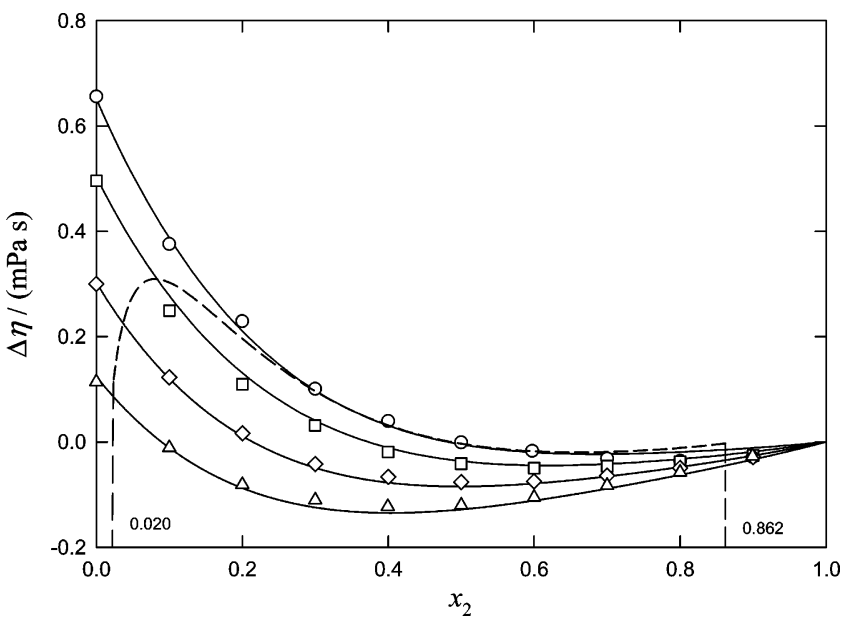

Figure 6. Viscosity, $\Delta \eta$, of the system water (1) + ethyl acetate (2) + ethanol (3) at $318.15 \mathrm{~K}$ following lines of constant $z=x_{3} / x_{1}$. The symbols represent experimental data, and the lines are calculated: $\bigcirc, z=0.43 ; \square$, $z=1.00 ; \diamond, z=2.33 ; \Delta, z=9.00 ;-$, eqs 5 and $6 ;---$, binodal curve..$^{22,23}$

Table 5. Viscosities, $\eta$, for the System Water (1) + Ethyl Acetate (2) + Ethanol (3) and Its Binary Constituents at $318.15 \mathrm{~K}$ and Atmospheric Pressure

\begin{tabular}{|c|c|c|c|c|c|}
\hline & & $\eta$ & & & $\eta$ \\
\hline$x_{1}$ & $x_{2}$ & $\mathrm{mPa} \cdot \mathrm{s}$ & $x_{1}$ & $x_{2}$ & $\mathrm{mPa} \cdot \mathrm{s}$ \\
\hline 0.0250 & 0.9750 & 0.362 & 0.2400 & 0.2004 & 0.650 \\
\hline 0.0496 & 0.9504 & 0.368 & 0.2101 & 0.3000 & 0.557 \\
\hline 0.0751 & 0.9250 & 0.373 & 0.1801 & 0.4000 & 0.498 \\
\hline 0.0999 & 0.9001 & 0.379 & 0.1501 & 0.5000 & 0.453 \\
\hline 0.1254 & 0.8746 & 0.385 & 0.1201 & 0.6000 & 0.420 \\
\hline 0.1000 & 0 & 0.848 & 0.0900 & 0.7000 & 0.396 \\
\hline 0.2000 & 0 & 0.951 & 0.0601 & 0.7999 & 0.376 \\
\hline 0.3001 & 0 & 1.003 & 0.0300 & 0.9000 & 0.362 \\
\hline 0.4036 & 0 & 1.090 & 0.0900 & 0.1000 & 0.685 \\
\hline 0.5002 & 0 & 1.168 & 0.0800 & 0.2000 & 0.577 \\
\hline 0.6000 & 0 & 1.243 & 0.0700 & 0.3001 & 0.510 \\
\hline 0.7000 & 0 & 1.298 & 0.0600 & 0.3994 & 0.460 \\
\hline 0.8007 & 0 & 1.293 & 0.0500 & 0.5000 & 0.425 \\
\hline 0.9000 & 0 & 0.977 & 0.0400 & 0.5999 & 0.402 \\
\hline 0 & 0.1000 & 0.624 & 0.0300 & 0.7000 & 0.387 \\
\hline 0 & 0.2000 & 0.535 & 0.0200 & 0.8000 & 0.374 \\
\hline 0 & 0.3007 & 0.471 & 0.0101 & 0.8991 & 0.367 \\
\hline 0 & 0.4000 & 0.432 & 0.1000 & 0.0900 & 0.704 \\
\hline 0 & 0.5000 & 0.412 & 0.2001 & 0.0800 & 0.781 \\
\hline 0 & 0.6003 & 0.387 & 0.2895 & 0.0710 & 0.850 \\
\hline 0 & 0.7006 & 0.374 & 0.4000 & 0.0600 & 0.946 \\
\hline 0 & 0.8000 & 0.364 & 0.5014 & 0.0499 & 1.034 \\
\hline 0 & 0.9000 & 0.360 & 0.6000 & 0.0400 & 1.128 \\
\hline 0.6300 & 0.1000 & 0.989 & 0.7000 & 0.0300 & 1.200 \\
\hline 0.5600 & 0.2000 & 0.814 & 0.8000 & 0.0200 & 1.204 \\
\hline 0.4900 & 0.3000 & 0.657 & 0.1000 & 0.2700 & 0.534 \\
\hline 0.4200 & 0.4000 & 0.567 & 0.2004 & 0.2399 & 0.593 \\
\hline 0.3500 & 0.4999 & 0.498 & 0.3001 & 0.2100 & 0.670 \\
\hline 0.2818 & 0.5974 & 0.454 & 0.4019 & 0.1794 & 0.761 \\
\hline 0.2101 & 0.6999 & 0.410 & 0.5002 & 0.1500 & 0.861 \\
\hline 0.1399 & 0.8001 & 0.377 & 0.6000 & 0.1200 & 0.987 \\
\hline 0.0703 & 0.8996 & 0.356 & 0.1000 & 0.4500 & 0.442 \\
\hline 0.4500 & 0.1000 & 0.890 & 0.2000 & 0.4000 & 0.493 \\
\hline 0.4000 & 0.2000 & 0.719 & 0.3005 & 0.3497 & 0.558 \\
\hline 0.3500 & 0.2999 & 0.609 & 0.4000 & 0.3000 & 0.630 \\
\hline 0.3000 & 0.4000 & 0.527 & 0.5006 & 0.2497 & 0.718 \\
\hline 0.2500 & 0.5000 & 0.473 & 0.6000 & 0.2000 & 0.827 \\
\hline 0.2000 & 0.6001 & 0.433 & 0.1002 & 0.6298 & 0.398 \\
\hline 0.1500 & 0.7000 & 0.405 & 0.1999 & 0.5601 & 0.439 \\
\hline 0.1000 & 0.7999 & 0.382 & 0.3000 & 0.4900 & 0.487 \\
\hline 0.0500 & 0.9000 & 0.362 & 0.4200 & 0.4000 & 0.551 \\
\hline 0.2701 & 0.1000 & 0.791 & & & \\
\hline
\end{tabular}

The calculation of the dynamic viscosity, $\eta$, is done using the equation

$$
\eta=K\left(\rho_{\mathrm{b}}-\rho\right) t
$$


Table 6. Coefficients of Equation 4 Fitted to the Excess Molar Volumes, $V_{\mathrm{m}}^{\mathrm{E}}$, and Viscosity Deviations, $\Delta \eta$, for the Binary Systems and of Equation 6 for the Ternary Systems

\begin{tabular}{|c|c|c|c|c|c|c|c|c|}
\hline system & & $B_{0}$ & $B_{1}$ & $B_{2}$ & $C_{1}$ & $C_{2}$ & $\sigma$ & AAD $\%$ \\
\hline \multicolumn{9}{|c|}{$T=298.15 \mathrm{~K}$} \\
\hline \multirow[t]{2}{*}{ water + ethyl acetate } & $V_{\mathrm{m}}^{\mathrm{E}} /\left(\mathrm{cm}^{3} \cdot \mathrm{mol}^{-1}\right)$ & -3.123 & -2.602 & - & 0.617 & - & 0.01 & 3.0 \\
\hline & $\Delta \eta /(\mathrm{mPa} \cdot \mathrm{s})$ & -0.059 & 0.038 & - & - & - & 0.00 & 5.8 \\
\hline \multirow[t]{2}{*}{ water + ethanol } & $V_{\mathrm{m}}^{\mathrm{E}} /\left(\mathrm{cm}^{3} \cdot \mathrm{mol}^{-1}\right)$ & -4.443 & - & - & -0.271 & - & 0.04 & 4.0 \\
\hline & $\Delta \eta /(\mathrm{mPa} \cdot \mathrm{s})$ & 3.861 & - & - & -1.271 & 0.554 & 0.03 & 2.7 \\
\hline \multirow[t]{2}{*}{ ethyl acetate + ethanol } & $V_{\mathrm{m}}^{\mathrm{E}} /\left(\mathrm{cm}^{3} \cdot \mathrm{mol}^{-1}\right)$ & 0.620 & - & - & -0.180 & - & 0.01 & 5.0 \\
\hline & $\Delta \eta /(\mathrm{mPa} \cdot \mathrm{s})$ & -0.995 & - & - & 0.652 & - & 0.00 & 1.0 \\
\hline \multirow[t]{2}{*}{ water + ethyl acetate + ethanol } & $V_{\mathrm{m}}^{\mathrm{E}} /\left(\mathrm{cm}^{3} \cdot \mathrm{mol}^{-1}\right)$ & 3.910 & -1170.750 & - & -450.302 & - & 0.02 & 3.0 \\
\hline & $\Delta h /(\mathrm{mPa} \cdot \mathrm{s})$ & -9.295 & -13.476 & - & - & 1.088 & 0.03 & 9.9 \\
\hline \multicolumn{9}{|c|}{$T=318.15 \mathrm{~K}$} \\
\hline \multirow[t]{2}{*}{ water + ethyl acetate } & $V_{\mathrm{m}}^{\mathrm{E}} /\left(\mathrm{cm}^{3} \cdot \mathrm{mol}^{-1}\right)$ & -4.298 & -3.493 & - & 0.210 & - & 0.01 & 4.0 \\
\hline & $\Delta \eta /(\mathrm{mPa} \cdot \mathrm{s})$ & -0.279 & -0.604 & -0.350 & - & - & 0.00 & 6.9 \\
\hline \multirow[t]{2}{*}{ water + ethanol } & $V_{\mathrm{m}}^{\mathrm{E}} /\left(\mathrm{cm}^{3} \cdot \mathrm{mol}^{-1}\right)$ & -4.134 & - & - & -0.290 & - & 0.05 & 5.0 \\
\hline & $\Delta \eta /(\mathrm{mPa} \cdot \mathrm{s})$ & 2.015 & 2.076 & 1.611 & - & - & 0.03 & 5.1 \\
\hline \multirow[t]{2}{*}{ ethyl acetate + ethanol } & $V_{\mathrm{m}}^{\mathrm{E}} /\left(\mathrm{cm}^{3} \cdot \mathrm{mol}^{-1}\right)$ & 1.115 & - & - & 0.007 & - & 0.02 & 6.0 \\
\hline & $\Delta \eta /(\mathrm{mPa} \cdot \mathrm{s})$ & -0.584 & 0.346 & -0.164 & - & - & 0.00 & 1.8 \\
\hline \multirow{2}{*}{ water + ethyl acetate + ethanol } & $V_{\mathrm{m}}^{\mathrm{E}} /\left(\mathrm{cm}^{3} \cdot \mathrm{mol}^{-1}\right)$ & 6.493 & 10.947 & - & - & 0.267 & 0.02 & 4.0 \\
\hline & $\Delta \eta /(\mathrm{mPa} \cdot \mathrm{s})$ & -2.925 & -0.191 & - & - & 0.554 & 0.01 & 9.8 \\
\hline
\end{tabular}

where $t$ is the falling time of the ball measured with the stopwatch. $K$ is a calibration constant, and $\rho_{\mathrm{b}}$ represents the density of the ball. The viscosity deviations, $\Delta \eta$, were obtained using the expression

$$
\Delta \eta=\eta-\sum x_{i} \eta_{i}
$$

where $\eta$ is the measured mixture viscosity and $\eta_{i}$ represents the pure component viscosity. The viscosity measurements were made for different compositions than those made for the density measurements because they were not performed simultaneously. For that reason, the density value needed to obtain the viscosity from eq 2 was calculated with eq 1 with a fitted equation for $V_{\mathrm{m}}^{\mathrm{E}}$.

In Tables 4 and 5 the viscosities, $\eta$, for the ternary system and constituent binaries at 298.15 and $318.15 \mathrm{~K}$ are shown.

The dependence of binary $V_{\mathrm{m}}^{\mathrm{E}}$ and $\Delta \eta$ on composition, $X_{i j}$, can be described by a flexible equation proposed by Myers and $\mathrm{Scott}^{7}$

$$
X_{i j}=x_{i} x_{j} \frac{\sum_{k=0}^{p} B_{k} z_{i j}^{k}}{1+\sum_{l=1}^{m} C_{l} z_{i j}^{l}}
$$

where $z_{i j}=x_{i}-x_{j} . B_{k}$ and $C_{l}$ are adjustable coefficients obtained from the fitting of eq 4 to $V_{\mathrm{m}}^{\mathrm{E}}$ or $\Delta \eta$.

The ternary data were correlated by the following equation

$$
X_{123}=\sum_{i<j} X_{i j}+X_{\mathrm{T}}
$$

where $X_{123}$ represents $\Delta V_{123}{ }^{\mathrm{E}}$ and $\Delta \eta_{123}$ for the ternary mixtures; $X_{i j}$ are functions for the same properties, obtained by fitting eq 4 to binary data; and $X_{\mathrm{T}}$ is a ternary contribution. To express $X_{\mathrm{T}}$, the Pando et al. expression ${ }^{8}$

$$
X_{\mathrm{T}}=x_{1} x_{2} x_{3}\left(\frac{B_{0}+B_{1} z_{13}+B_{2} z_{23}}{1+C_{1} z_{13}+C_{2} z_{23}}\right)
$$

was used. We have realized that three adjustable parameters were enough in eq 6 to reproduce the experimental data, and the use of more parameters does not improve the fitting.
Table 6 presents the coefficients $B_{k}$ and $C_{l}$ of eq 4 fitted to binary $V_{\mathrm{m}}^{\mathrm{E}}$ and $\Delta \eta$ data. The standard deviation, $\sigma$, and the average absolute deviation, AAD, of the corresponding fittings are also shown. The coefficients corresponding to the fitted eq 6 of the ternary data are also presented. The $\sigma$ and the AAD of the fittings are defined as usual

$$
\sigma=\left[\sum_{i=1}^{N} \frac{\left(X_{\text {calcd }}-X_{\text {exptl }}\right)_{i}^{2}}{N-m}\right]^{1 / 2}
$$

and

$$
\mathrm{AAD}=\frac{100}{N}\left[\sum_{i=1}^{N}\left|\left(X_{\text {calcd }}-X_{\text {expt } 1}\right) / X_{\text {exptl }}\right|_{i}\right]
$$

where $N$ and $m$ represent the number of experimental points and parameters, respectively, and $X$ stands for $V_{\mathrm{m}}^{\mathrm{E}}$ and $\Delta \eta$. In Figure 1a and $b$, we have plotted the experimental and fitted values of $V_{\mathrm{m}}^{\mathrm{E}}$ at $298.15 \mathrm{~K}$ for the binary systems water + ethanol and ethyl acetate + ethanol as a function of the composition of water and ester, respectively.

As can be seen, our data for the water + ethanol system are in good agreement with the literature values. For the ethyl acetate + ethanol system, our experimental values agree with those obtained by $\mathrm{Hu}$ et al. ${ }^{21}$ and show significant deviations from the data reported by Ortega et al. ${ }^{20}$ Plots of the excess molar volume at $318.15 \mathrm{~K}$ for the same binary systems are shown in Figure 1c and d. As the water + ethyl acetate system shows a short miscibility range, the data were not plotted although they have been used in correlation.

We have found in the literature excess molar volumes for ethyl acetate with methanol and with 1-propanol at 298.15 and $318.15 \mathrm{~K}^{1}{ }^{1}$ It is interesting to compare the relative magnitude of the $V_{\mathrm{m}}^{\mathrm{E}}$ values for the equimolar mixtures. At $298.15 \mathrm{~K}$, these systems have $V_{\mathrm{m}}^{\mathrm{E}}(x=0.5)$ values of $-0.07,+0.14$ (this work), and $+0.24 \mathrm{~cm}^{3} \cdot \mathrm{mol}^{-1}$ for methanol, ethanol, and 1-propanol, respectively. The corresponding values at 318.15 $\mathrm{K}$ are $-0.12,+0.28$ (this work), and $+0.30 \mathrm{~cm}^{3} \cdot \mathrm{mol}^{-1}$. The increasing $V_{\mathrm{m}}^{\mathrm{E}}$ values with increasing chain length of the alcohol may be explained by the reduction of intermolecular association between the molecules of the pure species (hydrogen bonding in alcohols and dipolar interactions in esters) and to a more difficult accommodation of the ester and alcohol molecules. 
In Figures 2 and 3, we compare experimental and calculated $V_{\mathrm{m}}^{\mathrm{E}}$ values for the ternary system at 298.15 and $318.15 \mathrm{~K}$ as a function of $x_{2}$ for different values of $z=x_{3} / x_{1}$. The values of the excess molar volume were obtained from eq 5 with the ternary contribution, $V_{\mathrm{T}}^{\mathrm{E}}$, defined by eq 6 . The experimental values of $V_{\mathrm{m}}^{\mathrm{E}}$ agree quite well with the calculated ones from the correlations. We have compared our ternary density data with those due to Resa et al. ${ }^{24}$ Considering $X=\rho$ in eq 8 with $\rho_{\text {calcd }}$ calculated from our correlations and $\rho_{\text {exptl }}$ being the experimental values of Resa et al., ${ }^{24}$ we obtained AAD values less than 0.1 $\%$ at the two temperatures.

The experimental and fitted values of the viscosity deviations at 298.15 and $318.15 \mathrm{~K}$ for the systems water + ethanol and ethyl acetate + ethanol as a function of the compositions of water and ester, respectively, are compared in Figure 4. In this figure, we have plotted also values taken from the literature. For the system water + ethanol, the literature values are in excellent agreement with our data. For the ethyl acetate + ethanol system, the data of Nikam et al. ${ }^{11}$ at $298.15 \mathrm{~K}$ deviate significantly from our values. It can be observed that the absolute values of $\Delta \eta$ decrease with the temperature for a fixed composition at both temperatures. In Figures 5 and 6, we compare the experimental and fitted viscosity deviations at the two temperatures studied for the ternary system as a function of $x_{2}$ for different values of $z=x_{3} / x_{1}$. The experimental and the calculated values of $\Delta \eta$ show good agreement.

\section{Conclusions}

Experimental viscosities and densities of water + ethyl acetate + ethanol were measured at 298.15 and $318.15 \mathrm{~K}$ and atmospheric pressure. The excess molar volume, $V_{\mathrm{m}}^{\mathrm{E}}$, and the viscosity deviations, $\Delta \eta$, have been well correlated using rational functions. Our $V_{\mathrm{m}}^{\mathrm{E}}$ binary data are in close agreement with those taken from the literature at $298.15 \mathrm{~K}$. For the viscosity deviation of both binary systems, the same conclusion can be drawn. The ternary $V_{\mathrm{m}}^{\mathrm{E}}$ and $\Delta \eta$ behavior is well described using additivity of binaries and a ternary term with only three coefficients.

Note Added after ASAP Publication. This paper was published ASAP on May 8, 2007. Data in Table 1 were changed. The updated paper was reposted on June 8, 2007.

\section{Literature Cited}

(1) Oswal, S. L.; Putta, S. S. R. Excess Molar Volumes of Binary Mixtures of Alkanols with Ethyl Acetate from 298.15 to 323.15 K. Thermochim. Acta 2001, 373, 141-152.

(2) Kijevcanin, M. L.; Ribeiro, I. S. A.; Ferreira, A. G. M.; Fonseca, I. M. A. Densities, Viscosities, and Surface and Interfacial Tensions of the Ternary Mixture Water + Ethyl Butyrate + Methanol at 303.15 K. J. Chem. Eng. Data 2003, 48, 1266-1270.

(3) Giro, F.; Gonçalves, M. F.; Ferreira, A. G. M.; Fonseca, I. M. A. Viscosity and Density Data of the System Water $+n$-Pentyl Acetate + Methanol. Calculations with a Modified Redlich-Kwong-Soave Equation of State. Fluid Phase Equilib. 2003, 204, 217-232.

(4) Lepori, L.; Matteoli, E. Excess Volumes of the Ternary System Ethanol + Tetrahydrofuran + Cyclohexane at 298.15 K. Fluid Phase Equilib. 1998, 145, 69-87.
(5) Lien, P.; Lin, H.; Lee, M. Excess Molar Enthalpies for the Binary Mixtures of Ethanol + Benzene, + Cyclohexane, + Propyl Acetate and 1-Propanol + Benzene, + Cyclohexane, + Heptane at 348.15 K. Fluid Phase Equilib. 2004, 215, 187-193.

(6) Marsh, K. N. A General Method for Calculating the Excess Gibbs Free Energy from Isothermal Vapor-Liquid Equilibria. J. Chem. Thermodyn. 1977, 9, 719-724.

(7) Myers, D. B.; Scott, R. L. Thermodynamic Functions For Nonelectrolyte Solutions. Ind. Eng. Chem. 1963, 55, 43-46.

(8) Pando, C.; Renuncio, J. A. R.; Calzon, J. A. G.; Christensen, J. J ; Izatt, R. M. Correlation and Prediction of Ternary Excess Enthalpy Data. J. Solution Chem. 1987, 16, 503-527.

(9) Sato, H.; Vematsu, M.; Watanabe, K. New International Skeleton Tables for the Thermodynamic Properties of Ordinary Water Substance. J. Phys. Chem. Ref. Data 1988, 17, 1439-1540.

(10) Kestin, J.; Sokolov, M.; Wakeham, A. Viscosity of Liquid Water in the Range $-8^{\circ} \mathrm{C}$ to $150^{\circ} \mathrm{C}$. J. Phys. Chem. Ref. Data 1978, 7, 941948 .

(11) Nikam, P. S.; Mahale, T. R.; Hasan, M. Density and Viscosity of Binary Mixtures of Ethyl Acetate with Methanol, Ethanol, Propan1-ol, Propan-2-ol, Butan-1-ol, 2-Methylpropan-1-ol, and 2-Methylpropan-2-ol at $(298.15,303.15$, and 308.15) K. J. Chem. Eng. Data 1996, 41, 1055-1058.

(12) Wilhoit, R. C.; Zwolinski, B. J. Physical and Thermodynamic Properties of Aliphatic Alcohols. J. Phys. Chem. Ref. Data 1973, 2, Suppl. No. 1.

(13) Canosa, J.; Rodriguez, A.; Tojo, J. Dynamic Viscosities of (Methyl Acetate or Methanol) with (Ethanol, 1-Propanol, 2-Propanol, 1-Butanol, and 2-Butanol) at 298.15 K. J. Chem. Eng. Data 1998, 43, 417421.

(14) Arce, A.; Blanco, A.; Soto, A.; Souza, P.; Vidal, J. Excess Volumes and Refractions and Liquid-Liquid Equilibria of the Ternary System Water + Ethanol + Hexyl Acetate. Fluid Phase Equilib. 1993, 87, $347-364$.

(15) Vargaftik, N. B. Handbook of Physical Properties of Liquids and Gases; Hemisphere Publishing: New York, 1975.

(16) Djujoputro, H.; Ismadji, S. Density and Viscosity Correlation for Several Common Fragance and Flavor Esters. J. Chem. Eng. Data 2005, 50, 727-731.

(17) Rauf, M. A.; Stewart, G. H.; Farhataziz. Viscosities and Densities of Binary Mixtures of 1-Alkanols from 15 to $55^{\circ} \mathrm{C}$. J. Chem. Eng. Data 1983, 28, 324-328.

(18) Arce, A.; Blanco, A.; Mendoza, J.; Soto, A. Densities, Refractive Indices, and Excess Molar Volumes of Water + Ethanol + 2-Methoxy methylpropane at 298.15 K. J. Chem. Eng. Data 1995, 40, 12851287.

(19) Benson, G. C.; Kiyohara, O. Thermodynamics of Aqueous Mixtures of Nonelectrolytes. 1. Excess Volumes of Water-n-Alcohol Mixtures at Several Temperatures. J. Solution Chem. 1980, 9, 791-804.

(20) Ortega, J.; Pena, J. A.; Paz-Andrade, M. I. Excess Molar Volumes of Binary Mixtures of Ethyl Acetate and Propyl Acetate with Normal Alkanols. Aust. J. Chem. 1986, 39, 1685-1690.

(21) Hu, J.; Tamura, K.; Murakami, S. Excess Thermodynamic properties of Binary Mixtures of Ethyl Acetate with Benzene, ethanol, and 2,2,2trifluoroethan-1-ol at 298.15 K. Fluid Phase Equilib. 1997, 134, 239253.

(22) Arce, A.; Alonso, L.; Vidal, I. Liquid-Liquid Equilibria of the Systems Ethyl Acetate + Ethanol + Water, Butyl Acetate + Ethanol + Water, and Ethyl Acetate + Butyl Acetate + Water. J. Chem. Eng. Jpn. 1999 $32,440-444$.

(23) Resa, J. M.; González, C.; Goenaga, J. M. Liquid-Liquid Equilibrium Diagrams of Ethanol + Water + (Ethyl Acetate or 1-Pentanol) at Several Temperatures. J. Chem. Eng. Data 2006, 51, 1300-1305.

(24) Resa, J. M.; González, C.; Goenaga, J. M.; Iglesias, M. Temperature Dependence of Excess Molar Volumes of Ethanol + Water + Ethyl Acetate. J. Solution Chem. 2004, 33, 169-198.

Received for review December 13, 2006. Accepted April 2, 2007.

JE600565M 